University of Nebraska - Lincoln

DigitalCommons@University of Nebraska - Lincoln

6-10-2002

\title{
Band filling and depletion through the doping of polyaniline thin films
}

B. $\mathrm{Xu}$

University of Nebraska-Lincoln

Jaewu Choi

University of Nebraska-Lincoln, jchoi@ece.eng.wayne.edu

A. N. Caruso

University of Nebraska-Lincoln, carusoan@umkc.edu

Peter A. Dowben

University of Nebraska-Lincoln, pdowben@unl.edu

Follow this and additional works at: https://digitalcommons.unl.edu/physicsdowben

Part of the Physics Commons

Xu, B.; Choi, Jaewu; Caruso, A. N.; and Dowben, Peter A., "Band filling and depletion through the doping of polyaniline thin films" (2002). Peter Dowben Publications. 19.

https://digitalcommons.unl.edu/physicsdowben/19

This Article is brought to you for free and open access by the Research Papers in Physics and Astronomy at DigitalCommons@University of Nebraska - Lincoln. It has been accepted for inclusion in Peter Dowben Publications by an authorized administrator of DigitalCommons@University of Nebraska - Lincoln. 


\title{
Band filling and depletion through the doping of polyaniline thin films
}

\author{
B. Xu, Jaewu Choi, ${ }^{a)}$ A. N. Caruso, and P. A. Dowben ${ }^{\text {b) }}$ \\ Department of Physics and Astronomy and the Center for Materials Research and Analysis (CMRA), Behlen \\ Laboratory of Physics, University of Nebraska-Lincoln, Lincoln, Nebraska 68588-0111
}

(Received 27 November 2001; accepted for publication 13 April 2002)

\begin{abstract}
The position of the molecular orbitals of polyaniline, relative to the Fermi level, shifts with the doping of polyaniline by both charge donor and charge acceptor species. Sodium as an electron donor, when added to the polyaniline system, results in an increase in electron population in the polyaniline bands but a decrease in density of states near $E_{F}$. This is evident from the consistent shifts toward the greater binding energy of the occupied as well as unoccupied molecular orbitals, the valence bands and conduction band edges and characteristic core levels. In the case of iodine doping, an electron acceptor, all the states were shifted toward smaller binding energy. These results provide compelling evidence that the molecular orbitals of vapor deposited polyaniline thin films are delocalized. () 2002 American Institute of Physics. [DOI: 10.1063/1.1484247]
\end{abstract}

Chemical shifts in photoemission have been recognized for more than three decades and core level photoemission chemical shifts are now commonly used to "fingerprint" the chemical state of many compounds and molecules. Systematic studies of combined valence and conduction band initial state chemical shifts, however, are rare. Such combined studies are essential for distinguishing between shifts in bands resulting from changes in metallicity and electron screening (electron spectroscopy final state effects) and charge transfer due to the addition of ligands, or alloying (initial state effects). The addition of ligands, doping or alloying necessary for significant chemical shifts of the valence band results in significant changes in the molecular orbitals (in molecules) and band structure (in solids) and can obscure changes in the valence band due to simple charge addition or subtraction. With insulators, doping can lead to a number of interesting complications. Changes in electron localization can lead to dramatic changes in photoemission screening ${ }^{1}$ and, as a consequence, photoemission final state effects rather than initial state chemical shifts can dominate the valence band. ${ }^{2}$ For wide band gap insulators, doping can lead to the formation of a density of states in the gap (Hubbard bands). These new bands can appear well away from the Fermi level (both above and below the Fermi level) as a result of the on site correlation energies. These Hubbard bands have been observed with alkali metal doping of a number of molecular systems that have a large gap between the highest occupied molecular orbital (HOMO) and the lowest unoccupied molecular orbital (LUMO) ${ }^{1,3,4}$ If the molecular orbitals are sufficiently delocalized, organic conductors provide an avenue by which to directly investigate systematic shifts of the valence and conduction band states as a result of charge transfer. For example, for single walled carbon nanotubes (CNTs), band structure calculations ${ }^{5-7}$ suggest that alkali metal doping leads to a transition from a semimetallic character to a more metallic character and modest band filling. The Fermi

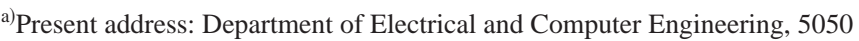
Anthony Wayne Dr., Wayne State University, Detroit, MI 48202.

b) Author to whom correspondence should be addressed; electronic mail: pdowben@unl.edu
}

level is positioned at higher energies relative to the graphite like band structure of the carbon nanotubes the greater the charge donation from the doping alkali metals.

Of the various candidate conducting polymers (e.g., polythiophene, polyacteylene, polypyrrole, polyaniline, etc. ${ }^{8}$ ), it has been demonstrated that polyaniline thin films can be produced by vapor deposition, ${ }^{9}$ and this is compatible with ultrahigh vacuum photoemission. ${ }^{10}$ These films can be grown very thin (100 $⿱$ or less) and are free of solvent contamination. Such films exhibit perpendicular resistance of only a few ohms at temperatures as low as $4 \mathrm{~K}$ and are thus free of surface charging in photoemission.

The polyaniline samples were prepared by vacuum deposition on Au-coated (500 $⿱$ ) silicon substrates in a preparation chamber vacuum continuous with the spectrometer vacuum systems. ${ }^{10}$ Angle-resolved photoemission spectroscopy experiments were performed on the $3 \mathrm{~m}$ toroidal grating monochromator at the Center for Advanced Microstructure and Devices (CAMD) ${ }^{10}$ The angle-resolved photoemission spectra reproduced here were taken with photon energy of $70 \mathrm{eV}$, the photon incident angle was $40^{\circ}(s+p$ polarized light) and the photoelectrons were collected normal to the surface. $\mathrm{He}(\mathrm{I})(21.2 \mathrm{eV})$ ultraviolet photoemission spectroscopy (UPS), angle-resolved inverse photoemission spectroscopy (IPES), and angle-resolved x-ray photoemission spectroscopy (XPS) were undertaken in a single UHV chamber. ${ }^{3,4}$ The Fermi level was determined from a tantalum foil in close contact with the sample substrate and the gold substrate.

A SEAS Na getter was used to sodium dope the polyaniline films, while iodine vapor was used to introduce iodine into the films. The $\mathrm{Na} 1 s$ to $\mathrm{C} 1 s$ x-ray photoemission intensity ratios show no emission angle dependence following $\mathrm{Na}$ deposition. This indicates that the sodium is uniformly distributed in the surface region of the vapor deposited polyaniline in a fashion similar to some other polymers. ${ }^{11}$ The result is similar in iodine doped polyaniline films. There is, therefore, no Schottky barrier formation like that reported for metal/conducting polymer heterojunctions prepared using polypyrrole, polyaniline, and polythiophene with low work function metals, e.g., $\mathrm{Al}, \mathrm{In}, \mathrm{Ag}, \mathrm{Mg} .{ }^{12-16}$ 


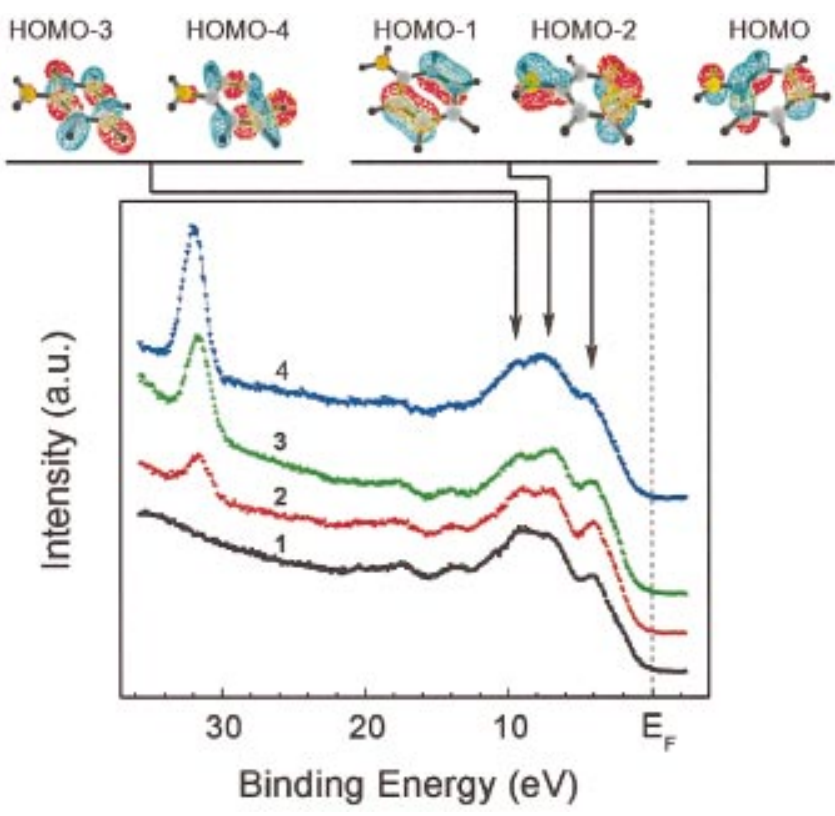

FIG. 1. (Color) Valence band evolution with increasing sodium doping of a polyaniline film (100 ̊ thick) using angle-resolved photoemission spectroscopy. The polyaniline film was deposited on a Au-coated (500 ̊) silicon substrate. The sodium doping level increases from bottom to top, as indicated by the intensity for the $\mathrm{Na} 2 p$ core level ( $30.5 \mathrm{eV}$ binding energy). Simplified schematics (based on aniline alone) of the HOMO, the overlapping HOMO-1 and HOMO-2, and the overlapping HOMO-3 and HOMO-4 photoemission features are shown in the inset.

The preferential configuration of native polyaniline includes an alternate tilt of one aromatic ring with respect to the next (the arene-like rings are not coplanar). ${ }^{10}$ In fact, we observe light polarization dependence of polyaniline films with thicknesses less than $50 \AA .^{10}$ Because the HOMO, HOMO-1, and HOMO-2 (schematically shown in Fig. 1) are enhanced with $s$-polarized light, although observed in both $p$ and $s$-polarized light, it is clear the phenylene rings (and the chain length) are mostly parallel with the surface, as discussed in detail elsewhere. ${ }^{10}$ With an increase in the thickness of polyaniline on gold, the phenylene rings tend to tilt out of the plane. ${ }^{10}$ The adoption of the polyaniline configuration with arene rings coplanar greatly enhances the conductivity of the polymer film along with a shift of the polyaniline molecular orbitals toward the Fermi level and an increase in the density of states in the vicinity of the Fermi level in thinner films. ${ }^{10,17}$ With the polyaniline aromatic rings tilted with respect to one another, the conductivity and density of states near $E_{F}$ decline. By growing slightly thicker films, we can reduce the density of states near the Fermi level and clearly demonstrate the different effects of doping of this ultrathin polyaniline film system with electron donors (sodium) and electron acceptors (iodine).

For the thicker films (about $100 \AA$ thick), some of the preferential orientation is lost and the density of states near the Fermi level is diminished. In such films, increasing the sodium doping (as seen from the increase in intensity of the shallow $2 p$ sodium core level at $30.5 \mathrm{eV}$ binding energy) does not increase the density of states near the Fermi level, but rather leads to a decrease in the density of states and a shift of the occupied molecular orbitals toward higher binding energies, as seen in Fig. 1.

This influence of electron donors from increased concenDownloaded 05 Sep 2006 to 129.93.16.206. Redistribution subject

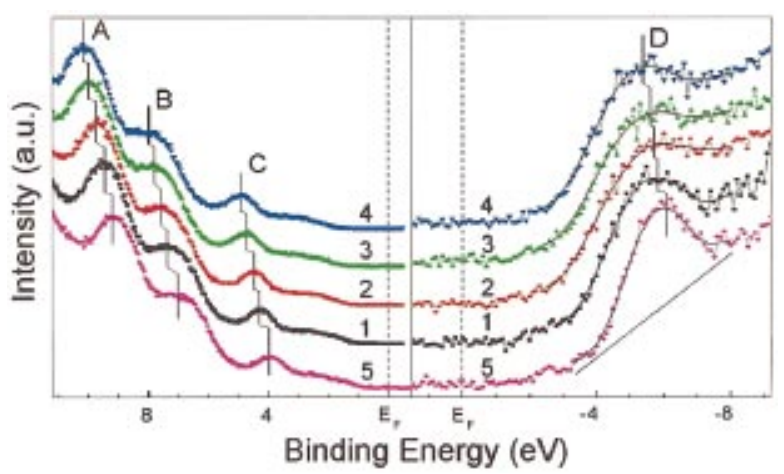

FIG. 2. (Color) Angle integrated ultraviolet photoemission spectra (left) and inverse photoemission (right) spectra (at normal incidence) of freshly evaporated polyaniline thin film $(100 \AA)$ as well as following sodium and iodine doping. Spectra 1 are from clean polyaniline film, spectra 2, 3, and 4 are from sodium-doped film with increased sodium exposure while spectra 5 are from iodine-doped film. Three photoemission features and one unoccupied inverse photoemission feature show similar binding energy shifts with doping (denoted as features A, B, C, and D, respectively).

trations of sodium, as well as electron subtraction from iodine doping of vapor deposited polyaniline on gold, is shown in Fig. 2. Both the occupied and unoccupied molecular orbitals of the freshly evaporated polyaniline thin films shift toward greater or smaller binding energies (with respect to the Fermi level) when doped with sodium or iodine, respectively. This is very different from sodium doped poly $(p \text {-pyridine })^{18}$ and other polymeric systems, ${ }^{1,4}$ where sodium doping induces new states in the otherwise empty band gap, but does resemble the increase in binding energies of the molecular orbitals observed with sodium doping of multiwalled carbon nanotubes. ${ }^{19}$

As seen in Fig. 2, the shifts in binding energies for valence bands or occupied molecular orbitals (features A, B, and $\mathrm{C}$ ) are the same and while the unoccupied levels (feature D) appear to exhibit a less significant shift in binding energy, the trend is very similar. Several fitting routines, with one illustrated in Fig. 2, were used to ascertain the shifts in the unoccupied levels, and all provided similar results. These shifts with a charge donor dopant or a charge acceptor dopant relative to the undoped vapor deposited polyaniline films are summarized in Fig. 3.

Within the picture of band filling, the introduction of

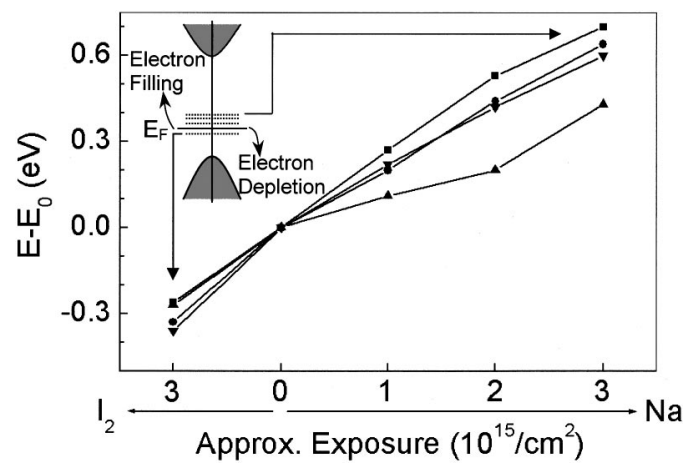

FIG. 3. Binding energy shifts for the occupied and unoccupied molecular orbital features of doped polyaniline, relative to undoped polyaniline, for each experimental deposition step. The symbols $\mathbf{\square}, \boldsymbol{\nabla}, \boldsymbol{\bullet}$, and $\boldsymbol{\Delta}$ correspond to features A, B, C, and D, respectively in Fig. 2. The approximate exposure is indicated for $\mathrm{Na}$ and $\mathrm{I}_{2}$. A schematic of electron filling and depletion is shown in the inset. 
extra electrons to the polyaniline system, through sodium atom doping, fills empty states near the Fermi level and increases the binding energies of all the molecular orbitals. The subtraction of electrons from the polyaniline system by doping with iodine atoms leads to a decrease in binding energy in all the bands. This concept is schematically shown in the inset of Fig. 3 and is consistent with our expectations of the effects of oxidation and protonation on a disordered onedimensional (1D) chain system. ${ }^{20,21}$ For films both thinner $(\sim 50 \AA)$ and thicker $(\sim 200 \AA)$ than those shown in Fig. 2, the shifts are qualitatively similar, but not as clearly evident. In the former case, this is due to the higher density of states in the vicinity of the Fermi energy and in the latter case it is more difficult to dope the film uniformly to significant levels by the methods employed here. The dopant atoms do not form a surface layer, but rather are isotropically distributed in the surface region probed by photoemission and inverse photoemission. This is clear from the absence of dramatic changes in the photoemission peak widths with doping (Fig. 2) and the angle-resolved XPS measurements mentioned above.

We thus conclude that with electron filling or depletion of the polyaniline bands, the molecular orbitals shift either toward greater (sodium) or smaller (iodine) binding energy with respect to the Fermi level. Since all the molecular orbitals (both occupied and unoccupied) shift very similar amounts with doping, we must conclude that molecular orbital shifts are dominated by charge transfer in the initial state and not by final state effects. ${ }^{2}$ The suppression of final state effects, in spite of changes in conductivity and the density of states in the vicinity of the Fermi level associated with such kinds of doping, suggests that the molecular orbitals are delocalized. The results are consistent with the expectations of doping another one-dimensional conductor: single walled carbon nanotubes. ${ }^{5-7}$ Since vapor deposited polyaniline is a $p$-type semiconductor, the simple picture of band filling or depletion explains why doping the material with electron donors makes this (and similar conducting polymers) more insulating, while doping with electron acceptors (halogens, perchlorate and the like) substantially increases conductivity, as is generally accepted. ${ }^{8}$ In the former case, the Fermi level is in a gap of the band structure, while in the latter case, the Fermi level resides well within an appreciable density of states of the band structure.

The authors thank A. Sokolov for his help in transport measurements of the polyaniline thin films. This work was supported by the Office of Naval Research, the Nebraska Research Initiative and the Petroleum Research Fund administered by the ACS.

${ }^{1}$ P. A. Dowben, Surf. Sci. Rep. 40, 151 (2000).

${ }^{2}$ J. E. Ortega, F. J. Himpsel, D. Li, and P. A. Dowben, Solid State Commun. 91, 807 (1994).

${ }^{3}$ D. N. McIlroy, C. Waldfried, T. McAvoy, J. Choi, P. A. Dowben, and D. Heskett, Chem. Phys. Lett. 264, 168 (1997).

${ }^{4}$ J. Choi, P. A. Dowben, C. N. Borca, S. Adenwalla, A. V. Bune, S. Ducharme, V. M. Fridkin, S. P. Palto, and N. Petukhova, Phys. Rev. B 59, 1819 (1999).

${ }^{5}$ Y. Miyamoto, A. Rubio, X. Blase, M. L. Cohen, and S. G. Louie, Phys. Rev. Lett. 74, 2993 (1995).

${ }^{6}$ E. G. Gal'pern, I. V. Stankevich, A. L. Chistykov, and L. A. Cherozantonskii, Chem. Phys. Lett. 214, 345 (1993).

${ }^{7}$ K. Esfarjani, A. A. Farajian, Y. Hashi, and Y. Kawazoe, Appl. Phys. Lett. 74, 79 (1999).

${ }^{8}$ A. B. Kaiser, Rep. Prog. Phys. 64, 1 (2001).

${ }^{9}$ R. V. Plank, N. J. DiNardo, and J. M. Vohs, Chem. Phys. Lett. 263, 33 (1996).

${ }^{10}$ J. Choi, M. Chipara, B. Xu, C. S. Yang, B. Doudin, and P. A. Dowben, Chem. Phys. Lett. 343, 193 (2001).

${ }^{11}$ B. Xu, J. Choi, C. N. Borca, A. V. Sorokin, P. A. Dowben, S. P. Palto, N. Petukhova, and S. G. Yudin, Appl. Phys. Lett. 78, 448 (2001).

${ }^{12}$ R. Gupta, S. C. K. Misra, B. D. Malhotra, N. N. Beladakere, and S. Chandra, Appl. Phys. Lett. 58, 51 (1991).

${ }^{13}$ S. Bandyopadhyay, A. Bhattacharyya, and S. K. Sen, J. Appl. Phys. 85, 3671 (1999)

${ }^{14}$ Y. Yang and A. J. Heeger, Appl. Phys. Lett. 64, 1245 (1994).

${ }^{15}$ M. Narasimhan, M. Hagler, V. Cammarata, and M. Thakur, Appl. Phys. Lett. 72, 1063 (1998).

${ }^{16}$ E. J. Lous, P. W. M. Blom, L. W. Molenkamp, and D. M. de Leeuw, Phys. Rev. B 51, 17251 (1995).

${ }^{17}$ P. E. Kornilovitch and A. M. Bratkovsky, Phys. Rev. B 64, 195413 (2001).

${ }^{18}$ G. Greczynski, N. Johansson, M. Lögdlund, L. A. A. Pettersson, W. R. Salaneck, L. E. Horsburgh, A. P. Monkman, D. A. dos Santos, and J. L. Brédas, J. Chem. Phys. 114, 4243 (2001).

${ }^{19}$ J. Choi, I. Samayoa, S. Lim, C. Jo, Y. Choi, Y. Lee, and P. A. Dowben, Phys. Lett. A (to be published).

${ }^{20}$ D. S. Galvão, D. A. dos Santos, B. Laks, C. P. de Melo, and M. J. Caldas, Phys. Rev. Lett. 63, 786 (1989).

${ }^{21}$ P. Phillips and H. Wu, Science 252, 1805 (1991). 